\title{
The Choice between Accrual and Real Earnings Management pre and post IPO of the VC-backed Companies
}

\author{
Dongxiang Lin* \\ School of Economics and Commerce, South China University \\ of Technology, Guangzhou, Guangdong, China \\ lindongxiang@foxmail.com
}

Keywords: Venture capital; Earnings management; IPO

\begin{abstract}
This paper mainly investigates the characteristic of earnings management pre and post IPO of the VC-backed companies from 2002 to 2014, based on accrual earnings management and real earnings management. The study finds that the VC-backed companies present more accrual and real earnings management than non VC-backed companies in the year before IPO and the main purpose is to promote the IPO. The VC-backed companies present more real earnings management than non VC-backed companies in the year of IPO and the main purpose is to facilitate the withdrawal through selling shares. The VC-backed companies don't present more accrual or real earnings management than non VC-backed companies in the yaer after IPO.
\end{abstract}

\section{投持股公司 IPO 前后应计盈余管理和真实盈余管理行为选择}

\author{
林东翔* \\ 华南理工大学经济与贸易学院, 广州, 广东, 中国 \\ lindongxiang@foxmail.com
}

关键词：风险投资；盈余管理；IP0

中文摘要：本文以 2002 年至 2014 年我国证券市场 IPO 公司为研究对象, 基于应计项目盈余 管理与真实活动盈余管理两种方式，实证探索风险投资持股公司在上市前后不同阶段的盈余 管理特征。实证结果如下:（1）IPO 前一年，有风险投资持股的公司相对无风险投资持股的 公司存在更高的应计盈余操控程度和真实盈余操控程度, 推动公司成功上市是其主要动机;

(2) IPO 当年，有风险投资持股的公司相对无风险投资持股的公司存在更高的真实盈余操控 程度，创造有利退出条件是重要原因;（3）IPO 后第一年，有风险投资持股的公司相对无风 险投资持股的公司并不存在更高的应计盈余操控程度或真实盈余操控程度。

\section{一、引言}

会计信息在国家进行宏观经济调控、促进社会资源的合理配置、资本市场监管等方面起 到了不可替代的作用，因此，公司 IPO 前后财务信息的真实性及盈余管理问题一直都是学术 界理论研究关注的重点，监管当局也在不断通过制定和完善各项规章制度，以加强对资本市 场上公司财务等相关信息披露的监管。而随着风险投资在中国的迅速兴起和发展, 很多处于 初创期或成长期的公司也得到了风险投资的支持，作为专业投资者，我国的风险投资机构是 会发挥监督认证作用，还是可能在追求公司业绩和自身利益等动机的推动下与公司通过盈余 管理来操纵利润,这将对 IPO 前后的财务信息质量产生新的影响, 从而出现了盈余管理问题。 近年来，我国的企业会计准则体系和监管机制也在不断完善，企业进行应计项目操控盈余的 
空间越来越小，公司逐渐倾向利用真实经营活动进行盈余管理。

目前国外针对风险投资与公司 IPO 盈余管理关系的相关研究相对丰富，但考虑到我国资 本市场尚不成熟、市场波动性大等宏观环境问题的存在, 国外风险投资的相关理论及经验成 果在我国特殊市场环境下的适用性有待进一步验证。而目前国内多集中在对创业板或中小板 公司 IPO 前后的盈余管理问题进行研究, 且研究结论不一, 因此也仍有待进一步完善。为了 全面地了解风投持股公司 IPO 前后各阶段的盈余管理特征，本文结合风投机构的投资特点， 基于应计项目盈余管理与真实活动盈余管理两种方式，对风投背景公司在上市前后不同阶段 的盈余管理问题进行跟踪研究。

\section{二、文献回顾}

风险投资的投资阶段主要为：笁选目标企业、辅助目标企业的经营管理并通过 IPO、兼 并收购等途径退出，对此投资过程，“监督理论” 认为：风险投资在对其所投资公司提供股权 融资等支持的同时，会监督公司的成长与发展，减少公司的盈余管理行为，使其在激励和监 督下获得比无风投背景公司更好的长期业绩，而风险投资则通过建立良好声誉获得长期利益。 监督理论最早是由 Barry et al（1990）提出，其发现风险投资机构可以利用其专业优势挑选出 发展潜力更好、盈利能力更强的公司，因此它们所投资的公司的整体质量相对同时期无风投 背景的上市公司质量会更高，即使它们投资了资质略差一些的公司，它们也会发挥其监督、 管理作用促进公司的成长与发展，使公司的整体质量得以提升，从而起到筛选和监督作用。

Morsfield and Tan（2006）以美国 1983 至 2001 年共 2630 家上市公司为研究对象，发现有风 险投资背景的上市公司的可操控利润相对无风险投资背景的上市公司明显较低, 有风险投资 背景的上市公司在 IPO 当年的盈余操控程度更低且在上市后业绩表现相对较好。Wongsunwai

（2013）通过研究风投机构声誉对公司盈余管理的影响，发现有更高声誉风险投资支持的公 司上市后有着更低的应计盈余操控程度和真实盈余操控程度, 财务重述的可能性也更低。国 内支持监督理论的研究有如陈祥有（2010）发现风险投资对中小板上市公司前一年的盈余管 理水平具有一定程度的抑制作用，佐证了认证监督理论。

以上研究都通过实证发现风险投资对上市公司的盈余管理行为具有一定的监督抑制作 用，但也有一些研究得出相反的结论，而支持了 “逐名理论”。“逐名理论” 认为：风险投资 会在追求短期业绩和自身利益等动机的驱使下与公司通过盈余管理操纵利润, 其所投资的公 司一般具有更高的盈余操控程度，以推动公司成功上市并在退出时获得更高的投资回报。逐 名理论最早是由 Gompers（1996）提出，其认为风险投资机构的参与可能会对其所投资的公 司产生负面影响。相对于成立时间较长的风投机构, 声誉较低的风投机构会有通过盈余操控 粉饰公司业绩，以尽早将公司包装上市，彰显其机构实力，提升风投机构声誉的 “逐名” 动 机。Cohen 和 Langberg（2009）研究获取风险投资是否有长期成本，结果发现有风险投资支 持的上市公司会计信息质量较低，且其会计信息可靠性与风险投资的持股比例和董事会席位 呈负相关关系, 另外风投机构作为上市公司股东, 会管理上市公司向资本市场所传递的信息, 从而获取短期利益。国内支持逐名理论的研究有如贾宁等（2011）以中小板的上市公司为样 本, 研究结果验证了 “逐名” 动机理论, 表明创投机构为尽快实现投资收益、证明自己的实 力会促使其投资企业过早上市，并且过度追求短期业绩。张莉和谈毅（2014）以 2009-2012 年创业板 355 家上市公司为研究对象, 实证结果显示有风投背景公司的盈余操控程度高于无 风投背景的公司，且联合风投个数越多，上市公司盈余操控程度越高。蔡宁（2015）研究发 现，风险投资支持的公司在 IPO 时和 IPO 后的应计盈余管理程度都高于无风投支持的公司, 主要出于推动企业上市、创造有利退出条件等利益动机。

总的来说，针对风险投资与盈余管理的国内外研究中，不同的样本，不同的研究方法， 研究结论也各不相同。国内研究多数以创业板或中小板的上市公司为研究对象, 而事实上, 风投投资遍布各个行业，对整个市场风险投资与公司盈余管理行为的关系有待进一步研究。 本文将以我国 2002 年至 2014 年的上市公司为研究对象, 运用多元线性回归模型, 探索有风 投背景的公司 IPO 前后各阶段的具体盈余管理特征表现。 


\section{三、理论分析与研究假设}

我国风险投资行业相比西方国家起步较晚，许多风投机构发展时间并不长，国内风险投 资会有强烈动机与公司通过盈余管理操纵利润，推动公司成功上市，从而获得更高的投资回 报并迅速建立行业声誉; 另外, 通过调增上市前一年的利润, 公司可以获得更高的整体估值, 并从股票的发行中得到更多的融资，有利于公司以后的发展。因此，有风险投资支持的公司 可能会进行更为显著的正向盈余管理, 且公司可能会充分利用应计盈余管理和真实盈余管理 两种方式进行利润操控。基于以上理论分析，本文提出以下假设:

假设 1: IPO 前一年，有风险投资持股的公司相对无风险投资持股的公司存在更高的应计 盈余操控程度和真实盈余操控程度。

风投机构的股权锁定期大多数为 1 年，因此，公司上市当年为风投机构投资退出的铺垫 期, 出于股票解禁后退出的考虑, 风投机构有动机促进公司的盈余管理行为, 使公司账面上 表现出更好的业绩，从而引导市场对公司股票作出更好的预期，以获得更高的市场定价，创 造有利的退出时机。此外，考虑到应计项目操控的盈余管理通常只会改变盈余在不同会计期 间的分布，而不会改变各期间的盈余总额，若前期通过应计盈余管理调增利润，在随后几年 必然出现盈余反转, 影响公司未来的会计利润表现, 不利于风投机构的退出, 而前面分析到, 有风险投资支持的公司在上市前一年已充分利用应计盈余管理方式进行利润操纵，因此，有 风险投资支持的公司在上市当年可能偏向于使用真实盈余管理方式。基于以上理论分析，本 文提出以下假设:

假设 2: IPO 当年，有风险投资持股的公司相对无风险投资持股的公司存在更高的真实盈 余操控程度。

公司上市后第一年，多数风险投资所持有的股票已解禁，此时风险投资会等待合适的时 机从二级市场中退出, 因此可能还会存在正向盈余管理的动机, 以创造有利退出条件, 但因 前期有风险投资支持的公司为达到上市目的已充分利用应计盈余管理和真实盈余管理两种策 略进行利润操控, 且无论应计盈余管理还是真实盈余管理都不是可持续使用的管理策略; 另 外，公司上市获得资金后，即使是有风险投资支持的公司也会出于避税动机，减少公司的盈 余管理行为。因此，在多种动机的共同作用下，有风投支持的公司在上市后第一年可能并不 存在更高的盈余操控程度。基于以上理论分析，本文提出以下假设：

假设 3: IPO 后第一年，有风险投资持股的公司相对无风险投资持股的公司并不存在更高 的应计盈余操控程度或真实盈余操控程度。

\section{四、研究设计}

\section{(一) 研究数据}

本文以 2002 年至 2014 年我国证券市场 IPO 公司为研究对象，风险投资数据及上市公司 财务数据主要来源于 CVSource 数据库和 Wind 数据库。剔除 IPO 前后财务数据不全的上市公 司，另外，考虑到金融行业的特殊性，对金融行业的上市公司数据也予以剔除，最终得到的 研究样本共 1436 个，其中有风险投资支持的公司共 515 个，占比 $35.9 \%$, 无风险投资支持的 公司共 921 个，占比 $64.1 \%$ 。

\section{（二）应计盈余管理模型}

应计项目操控盈余管理主要是企业管理当局在遵循会计准则的基础上, 通过对会计政策 选择、会计估计变更来控制或调整会计收益信息。会计政策选择如收益与费用确认时点的选 择、发出存货的计价方法、折旧方法选择等，会计估计的变更如固定资产折旧、无形资产摊 销年限和残值的估计, 坏账准备的计提, 预计负债的估计等。这种盈余管理方式通常只会改 变盈余在不同会计期间的分布，而不会改变各期间的盈余总额和公司的经营现金净流量。

从现有研究文献来看, 衡量盈余管理的方法很多, 但修正的 Jones 模型应用最为广泛, 国外研究表明修正的 Jones 模型估算盈余管理水平最佳。模型如下:

(1) 经营应计项目 (TACC) 的计算 
$\mathrm{TACC}_{\mathrm{t}}=\mathrm{EARN}_{\mathrm{t}}-\mathrm{CFO}_{\mathrm{t}}$

其中, $\mathrm{TACC}_{\mathrm{t}}$ 为公司第 $\mathrm{t}$ 期的总体应计利润, $\mathrm{EARN}_{\mathrm{t}}$ 为公司第 $\mathrm{t}$ 期的净利润, $\mathrm{CFO}_{\mathrm{t}}$ 为公司第 $\mathrm{t}$ 期的经营活动净现金流量。

(2) 经营应计项目不可操控部分 (NDAC) 的计算

$\mathrm{TACC}_{\mathrm{t}} / \mathrm{TA}_{\mathrm{t}-1}=\mathrm{a}_{0} / \mathrm{TA}_{\mathrm{t}-1}+\mathrm{a}_{1}\left(\triangle \mathrm{SALES}_{\mathrm{t}}-\triangle \mathrm{AR}_{\mathrm{t}}\right) / \mathrm{TA}_{\mathrm{t}-1}+\beta_{0} \mathrm{PPE}_{\mathrm{t}} / \mathrm{TA}_{\mathrm{t}-1}+\mathrm{e}_{\mathrm{t}}$

其中, $\mathrm{TA}_{\mathrm{t}-1}$ 为公司第 $\mathrm{t}-1$ 期的总资产, $\triangle \mathrm{SALES}_{\mathrm{t}}$ 为公司 $\mathrm{t}$ 期与 $\mathrm{t}-1$ 期营业收入的变化额, $\triangle \mathrm{AR}_{\mathrm{t}}$ 为公司 $\mathrm{t}$ 期与 $\mathrm{t}-1$ 期应收账款变化额, $P \mathrm{PE}_{\mathrm{t}}$ 为固定资产净值。通过估计式中各参数, 进 而计算出被接受变量 $\mathrm{TACC}_{\mathrm{t}} / \mathrm{TA}_{\mathrm{t}-1}$ 在样本区间的拟合值, 即可得到经营应计项目不可操控部 分的估计值:

$\mathrm{NDAC}_{\mathrm{t}}=\mathrm{a}_{0} / \mathrm{TA}_{\mathrm{t}-1}+\mathrm{a}_{1}\left(\triangle \mathrm{SALES}_{\mathrm{t}}-\triangle \mathrm{AR}_{\mathrm{t}}\right) / \mathrm{TA}_{\mathrm{t}-1}+\beta_{0} \mathrm{PPE}_{\mathrm{t}} / \mathrm{TA}_{\mathrm{t}-1}$

(3) 经营应计项目可操控部分 (DAC) 的计算

可操控经营应计项目由公司总的经营应计项目减去估计出的经营应计项目不可操控部分 得到:

$\mathrm{DAC}_{\mathrm{t}}=\mathrm{TACC}_{\mathrm{t}} / \mathrm{TA}_{\mathrm{t}-1}-\mathrm{NDAC}_{\mathrm{t}}$

\section{（三）真实盈余管理模型}

本文借鉴 Roychowdhury（2006）的研究方法，从三个方面衡量真实活动操控：第一，销 售操控：企业可通过放宽销售条件限制、信用条件和增加销售折扣等增加销售收入; 第二, 生产操控：企业可通过增加当年产量以降低单位产品固定成本，进而降低单位产品成本，以 达到增加利润等目的; 第三, 酌量性费用操控: 企业可通过减少研发支出、广告宣传支出等 相关费用进而影响当年利润。三种操控方式各表现出不同的特征, 销售操控会减少单位销售 带来的经营现金流, 生产操控会增加当年的总生产成本, 酌量性费用操控会减少当年的销售 费用和管理费用，可分别用异常经营活动净现金流、异常产品成本和异常酌量性费用来衡量。

本文将通过线性回归估算出经营活动净现金流、产品成本和酌量性费用的正常值，然后 利用三个项目的实际数减去其正常值, 得出异常值, 具体计算过程如下:

(1) 经营活动净现金流正常部分计算

根据 Dechow 等 (1995)，经营活动现金流 CFO 和销售额存在如下线性关系， 通过回归可计算正常现金流 NCFO :

$\mathrm{CFO}_{\mathrm{t}} / \mathrm{TA}_{\mathrm{t}-1}=\mathrm{a}_{0}+\mathrm{a}_{1} / \mathrm{TA}_{\mathrm{t}-1}+\beta_{0} \mathrm{SALES}_{\mathrm{t}} / \mathrm{TA}_{\mathrm{t}-1}+\beta_{1} \triangle \mathrm{SALES}_{\mathrm{t}} / \mathrm{TA}_{\mathrm{t}-1}+\mathrm{e}_{\mathrm{t}}$

异常经营活动净现金流定义如下:

$\mathrm{EM}_{-} \mathrm{CFO}_{\mathrm{t}}=\mathrm{CFO}_{\mathrm{t}} / \mathrm{TA}_{\mathrm{t}-1}-\mathrm{NCFO}_{\mathrm{t}} / \mathrm{TA}_{\mathrm{t}-1}$

(2) 产品成本正常部分计算

产品成本等于当年销售产品成本加上当年存货的变动额, 销售产品成本 $\mathrm{COGS}_{\mathrm{t}}$ 和销售额存在如下线性关系:

$\mathrm{COGS}_{\mathrm{t}} / \mathrm{TA}_{\mathrm{t}-1}=\mathrm{a}_{0}+\mathrm{a}_{1} / \mathrm{TA}_{\mathrm{t}-1}+\beta_{0} \mathrm{SALES}_{\mathrm{t}} / \mathrm{TA}_{\mathrm{t}-1}+\mathrm{e}_{\mathrm{t}}$

存货变动额和当期及上期销售变动额存在如下线性关系:

$\triangle \mathrm{INV}_{\mathrm{t}} / \mathrm{TA}_{\mathrm{t}-1}=\mathrm{a}_{0}+\mathrm{a}_{1} / \mathrm{TA}_{\mathrm{t}-1}+\beta_{0} \triangle \mathrm{SALES}_{\mathrm{t}} / \mathrm{TA}_{\mathrm{t}-1}+\beta_{1} \triangle \mathrm{SALES}_{\mathrm{t}-1} / \mathrm{TA}_{\mathrm{t}-1}+\mathrm{e}_{\mathrm{t}}$

所以产品成本 PROD 与销售额存在如下线性关系, 通过回归可计算正常产品成本 NPROD:

$\mathrm{PROD}_{\mathrm{t}} / \mathrm{TA}_{\mathrm{t}-1}=\mathrm{a}_{0}+\mathrm{a}_{1} / \mathrm{TA}_{\mathrm{t}-1}+\beta_{0} \mathrm{SALES}_{\mathrm{t}} / \mathrm{TA}_{\mathrm{t}-1}+\beta_{1} \triangle \mathrm{SALES}_{\mathrm{t}} / \mathrm{TA}_{\mathrm{t}-1}+$

$\beta_{2} \triangle$ SALES $_{\mathrm{t}-1} / \mathrm{TA}_{\mathrm{t}-1}+\mathrm{e}_{\mathrm{t}}$

异常产品成本定义如下:

EM_PROD $=\mathrm{PROD}_{\mathrm{t}} / \mathrm{TA}_{\mathrm{t}-1}-\mathrm{NPROD}_{\mathrm{t}} / \mathrm{TA}_{\mathrm{t}-1}$

（3）酌量性费用正常部分计算

酌量性费用 DISP 和销售额存在如下线性关系，通过回归可计算正常酌量性费用 NDISP :

$\mathrm{DISP}_{\mathrm{t}} / \mathrm{TA}_{\mathrm{t}-1}=\mathrm{a}_{0}+\mathrm{a}_{1} / \mathrm{TA}_{\mathrm{t}-1}+\beta_{0}$ SALES $_{\mathrm{t}} / \mathrm{TA}_{\mathrm{t}-1}+\mathrm{e}_{\mathrm{t}}$

异常酌量性费用定义如下:

EM_DISP $_{t}=\mathrm{DISP}_{\mathrm{t}} / \mathrm{TA}_{\mathrm{t}-1}-\mathrm{NDISP}_{\mathrm{t}} / \mathrm{TA}_{\mathrm{t}-1}$ 
因企业可能同时使用这三种方式进行真实盈余管理，所以本文设计了真实盈余管理的总 体计量指标:

$\mathrm{EM}_{-} \mathrm{PROXY} \mathrm{t}_{\mathrm{t}}=\mathrm{EM} \_\mathrm{PROD} \mathrm{D}_{\mathrm{t}}-\mathrm{EM} \_\mathrm{CFO} \mathrm{t}_{\mathrm{t}}-\mathrm{EM}_{-} \mathrm{DISP} \mathrm{t}_{\mathrm{t}}$

当企业采用真实盈余管理向上做大利润时，将表现出更低的经营活动净现金流、更高的 生产成本和更低的酌量性费用, 在扣除不可操控的正常部分后, 企业将具有更低的异常净现 金流、更高的异常产品生产成本和更低的异常酌量性费

用，此时总体计量指标 EM_PROXY 为正，反之为负。

\section{（四）变量设计}

被解释变量：DAC 为可操控性应计利润; EM_CFO 为异常经营活动净现金流, EM_PROD 为 异常生产成本, EM_DISP 为异常酌量性费用, EM_PROXY 为真实盈余管理水平。

解释变量：VC 为风投背景虚拟变量，若有风投背景，该变量取 1 , 否则取 0 。

控制变量: 本文参考 Roychowdhury 和李增福等的做法，引入可能影响盈余管理的相关因 素, 具体包括: SIZE 为公司规模, 以年末总资产的自然对数计量。DEBT 为财务杆杜, 以总负 债/总资产计量。GROW 为营业收入增长率, 以营业收入变化的百分比计量。ROA 为资产收益率, 以净利润与期初和期末平均资产总额之比计量。TURN 为资产周转率，以主营业务收入除以年 末总资产计量。 $\mathrm{RC}$ 为实际控制人类型, 若实际控制人为国有, 该变量取 1 , 否则取 0 。 UW 为 主承销商声誉虚拟变量, 若主承销商为当年排名前十的券商, 该变量取 1 , 否则取 0 , 主承销 商的排名数据来源于中国证券业协会每年公布的证券公司承销业务排名情况; AUDIT 为会计 师事务所声誉虚拟变量, 若年度审计报告为当年排名前十的会计师事务所出具, 该变量取 1 , 否则取 0 , 会计师事务所排名数据来源于中国注册会计师协会每年公布的会计师事务所综合 评价前百家信息。

\section{五、实证结果分析 \\ （一）差异性检验}

表 1 为盈余管理变量差异性检验结果，从中可以发现，有风险投资支持和无风险投资支 持的上市公司在上市前后各个阶段的盈余操控程度有所不同, DAC 的均值差异均为正数, 表 明有风险投资支持的上市公司通过应计项目进行盈余操控的平均程度高于无风投背景的上市 公司, 该均值差异在上市前一年和上市当年均具有显著性。EM_PROXY 的均值差异同样都为正 数, 表明有风投背景的上市公司通过真实活动进行盈余操控的平均程度高于无风投背景的上 市公司，该均值差异在上市前一年、上市当年及上市后第一年都具有显著性，由此初步说明 出于各种利益动机，有风险投资支持的公司相对无风险投资支持的上市公司在上市前后不同 阶段可能存在较高的的应计盈余操控程度或真实盈余操控程度, 下文将通过实证分析做进一 步研究。 
表 1 盈余管理变量差异性检验

\begin{tabular}{|c|c|c|c|c|c|c|c|}
\hline & & 均值 & 标准差 & 均值 & 标准差 & 均值差异 & $\mathrm{t}$ 值 \\
\hline \multicolumn{2}{|c|}{ 有无风投背景 } & \multicolumn{2}{|c|}{ 有风投背景 } & \multicolumn{2}{|c|}{ 无风投背景 } & & \\
\hline \multirow{3}{*}{ DAC } & IPO-1 & 0.0156 & 0.0928 & -0.0102 & 0.0862 & $0.0258 * * *$ & -4.2720 \\
\hline & IPO & 0.0136 & 0.14445 & -0.0090 & 0.1319 & $0.0226^{* *}$ & -2.3985 \\
\hline & IPO+1 & 0.0039 & 0.0641 & -0.0018 & 0.0834 & 0.0057 & -0.9936 \\
\hline \multirow{3}{*}{ EM_PROXY } & IPO-1 & 0.0322 & 0.2909 & -0.0209 & 0.3005 & $0.0531 * * *$ & -2.6351 \\
\hline & IPO & 0.0314 & 0.3452 & -0.0208 & 0.3462 & $0.0522 * *$ & -2.1893 \\
\hline & IPO+1 & 0.0225 & 0.1645 & -0.0104 & 0.1961 & $0.0329 * *$ & -2.3691 \\
\hline \multirow{3}{*}{ EM_CFO } & IPO-1 & -0.0147 & 0.1094 & 0.0096 & 0.0997 & $-0.0243 * * *$ & 3.4499 \\
\hline & IPO & -0.0178 & 0.1540 & 0.0118 & 0.1451 & $-0.0296 * * *$ & 2.8817 \\
\hline & IPO+1 & -0.0114 & 0.0729 & 0.0053 & 0.0891 & $-0.0167 * * *$ & 2.6578 \\
\hline \multirow{3}{*}{ EM_PROD } & IPO-1 & 0.0123 & 0.1497 & -0.0080 & 0.1505 & -0.1382 & -1.9913 \\
\hline & IPO & 0.0067 & 0.1630 & -0.0046 & 0.1690 & 0.0113 & -0.9726 \\
\hline & IPO+1 & 0.0066 & 0.0763 & -0.0031 & 0.0934 & 0.0097 & -1.4735 \\
\hline \multirow{3}{*}{ EM_DISP } & IPO-1 & -0.0052 & 0.1091 & 0.0034 & 0.1107 & -0.0086 & 1.1375 \\
\hline & IPO & -0.0069 & 0.1104 & 0.0046 & 0.1128 & -0.0115 & 1.4889 \\
\hline & IPO+1 & -0.0045 & 0.0580 & 0.0021 & 0.0613 & -0.0066 & 1.4615 \\
\hline
\end{tabular}

注：IP0-1 表示 IP0 前一年，IP0 表示 IP0 当年，IP0+1 表示 IP0 后第一年

\section{（二）多元线性回归分析}

表 2 反映了 IP0 上一年是否有风投持股与盈余管理关系的回归结果，其中，VC 的系数在 以 DAC 为因变量的回归结果中显著为正, 在以 EM_PROXY 为因变量的回归结果中同样显著为正, 表明有风投持股的上市公司在上市前一年存在更高的的应计盈余操控程度和真实盈余操控程 度，实证结果支持了理论假设 1 。

承销商声誉 UW 在两个模型中的系数为正, 但不显著, 表明国内综合实力强的承销商对拟 上市公司在上市前一年的盈余管理行为并没有起到更好的监督作用。审计机构声誉 AUDIT 在 两个模型中的系数为负, 但均不显著, 表明审计机构对拟上市公司的盈余管理行为起到监督 作用，但审计机构的声誉高低与监督作用大小不相关。 
表 $2 \quad I P O$ 上一年风投持股与盈余管理关系多元线性回归结果

\begin{tabular}{lrrrrr}
\hline & \multicolumn{1}{l}{ DAC } & EM_CFO & EM_PROD & EM_DISP & EM_PROXY \\
\hline VC & $0.0186^{* * *}$ & $-0.0244^{* * *}$ & $0.0302^{* * * *}$ & $-0.0150^{* * *}$ & $0.0696^{* * *}$ \\
GROW & $0.0005^{* * *}$ & $-0.0004^{* *}$ & $0.0007^{* * *}$ & $0.0005^{* * *}$ & 0.0006 \\
RC & $-0.0205^{* *}$ & $0.0296^{* * *}$ & -0.0174 & 0.0095 & $-0.0565^{* *}$ \\
ROA & 0.0001 & $0.0102^{* * *}$ & $-0.0129^{* * *}$ & $0.0052^{* * *}$ & $-0.0282^{* * *}$ \\
SIZE & 0.0039 & 0.0045 & $-0.0150^{* * *}$ & $0.0095^{* *}$ & $-0.0290^{* *}$ \\
TURN & -0.0071 & $-0.0120^{*}$ & 0.0011 & 0.0006 & 0.0125 \\
UW & 0.0012 & -0.0026 & 0.0141 & -0.0005 & 0.0172 \\
AUDIT & -0.0066 & 0.0036 & -0.0100 & 0.0039 & -0.0176 \\
DEBT & -0.0004 & $0.0012^{* * *}$ & $0.0008^{*}$ & 0.0000 & -0.0004 \\
\hline
\end{tabular}

注: *表示在 $10 \%$ 的显著性水平下显著, $* *$ 表示在 $5 \%$ 的显著性水平下显著, $* * *$ 表示在 $1 \%$ 的显著性水平下显著

表 3 反映了 IPO 当年是否有风投持股与盈余管理关系的回归结果，其中，VC 在 DAC 模型 中的系数为正但不显著, 在 EM_PROXY 中系数显著为正, 表明有风投持股的公司在上市当年存 在更高的真实盈余操控程度，实证结果支持了理论假设 2 。

承销商声誉 UW 在实证模型中都不显著, 表明国内综合实力强的承销商对上市公司在上市 当年的盈余管理行为并没有起到更好的监督作用。审计机构声誉 AUDIT 在两个模型中也都不 显著, 表明国内综合实力强的会计师事务所对上市公司在上市当年的盈余管理行为同样没有 起到更好的监督作用，这与上市前一年的表现基本一致。

表 3 IPO 当年风投持股与盈余管理关系多元线性回归结果

\begin{tabular}{lrrrrr}
\hline & \multicolumn{1}{c}{ DAC } & \multicolumn{1}{c}{ EM_CFO } & EM_PROD & \multicolumn{1}{c}{ EM_DISP } & EM_PROXY \\
\hline VC & 0.0150 & $-0.0292^{* * *}$ & $0.0283^{* * *}$ & $-0.0196^{* * *}$ & $0.0772^{* * *}$ \\
GROW & $0.0010^{* * *}$ & -0.0004 & $0.0009^{* * * *}$ & $0.0005^{* * *}$ & 0.0007 \\
RC & $-0.0380^{* * *}$ & $0.0441^{* * *}$ & 0.0005 & -0.0147 & -0.0289 \\
ROA & -0.0005 & $0.0167 * * *$ & $-0.0237^{* * *}$ & $0.0073^{* * *}$ & $-0.0478^{* * *}$ \\
SIZE & 0.0086 & -0.0051 & $-0.0151^{* *}$ & 0.0042 & -0.0142 \\
TURN & 0.0215 & -0.0185 & $0.0288^{* *}$ & -0.0160 & $0.0633^{* *}$ \\
UW & -0.0012 & 0.0023 & 0.0069 & 0.0008 & 0.0038 \\
AUDIT & -0.0118 & 0.0026 & -0.0005 & 0.0045 & -0.0076 \\
DEBT & $-0.0009 * *$ & 0.0003 & $0.0015^{* * *}$ & -0.0004 & $0.0016^{*}$ \\
\hline
\end{tabular}

注: *表示在 $10 \%$ 的显著性水平下显著, $* *$ 表示在 $5 \%$ 的显著性水平下显著, $* * *$ 表示在 $1 \%$ 的显著性水平下显著

表 4 反映了 IP0 后第一年是否有风投持股与盈余管理关系的回归结果，其中，VC 在 DAC 模型和 EM_PROXY 模型中系数为正, 但都不显著, 表明有风投持股的公司相对无风投持股的公 司并不存在更高的应计盈余操控程度或真实盈余操控程度，实证结果支持了理论假设 3 。

承销商声誉 UW 在实证模型中都不显著, 表明国内综合实力强、声誉高的承销商在上市 
后持续督导期对上市公司的盈余管理行为并没有起到更好的监督作用。审计机构声誉 AUDIT 在两个模型中系数显著为负, 表明审计机构对公司上市后第一年的盈余管理行为起到监督作 用，且综合实力强的公司其审计越严格。

表 4 IPO 后第一年风投持股与盈余管理关系多元线性回归结果

\begin{tabular}{lrrrrr}
\hline & \multicolumn{1}{c}{ DAC } & \multicolumn{1}{c}{ EM_CFO } & EM_PROD & EM_DISP & EM_PROXY \\
\hline VC & 0.0072 & -0.0082 & 0.0037 & -0.0043 & 0.0162 \\
GROW & $0.0006^{* * *}$ & $-0.0005^{* * *}$ & $0.0005^{* * *}$ & $0.0002^{* *}$ & $0.0008^{* * *}$ \\
RC & $-0.0284^{* * *}$ & $0.0325^{* * *}$ & -0.0038 & -0.0024 & $-0.0339^{* *}$ \\
ROA & 0.0000 & $0.0111^{* * *}$ & $-0.0140^{* * *}$ & $0.0034^{* * *}$ & $-0.0284^{* * *}$ \\
SIZE & 0.0009 & -0.0046 & 0.0035 & -0.0030 & 0.0111 \\
TURN & -0.0082 & 0.0027 & -0.0122 & 0.0072 & -0.0220 \\
UW & -0.0077 & 0.0064 & -0.0072 & 0.0004 & -0.0140 \\
AUDIT & $-0.0153^{* * *}$ & $0.0130^{* *}$ & $-0.0147 * *$ & 0.0058 & $-0.0335^{* * *}$ \\
DEBT & $0.0006 * * *$ & 0.0002 & 0.0001 & 0.0000 & 0.0000 \\
\hline
\end{tabular}

注: *表示在 $10 \%$ 的显著性水平下显著，**表示在 $5 \%$ 的显著性水平下显著, $* * *$ 表示在 $1 \%$ 的显著性水平下显著

\section{六、稳健性检验}

出于稳健性考虑，本文从以下两方面对研究结论进行检验。

（1）本文采用了修正的琼斯模型度量应计盈余操控程度，在此基础上，本文另外采用了 扩展的琼斯模型度量应计盈余操控程度, 即在修正的琼斯模型基础上引入总资产收益率 ROA 作为控制变量，对本文研究结论做进一步验证，实证发现，研究结论与本文一致。

（2）本文采用虚拟变量表示公司是否有风投背景，在此基础上，本文还另外采用风投机 构持股比例变量 VCS 代替虚拟变量进行实证研究，无风投背景公司 VCS 设为 0 ，有风险投资 持股的公司变量 VCS 取风投持股总比例。研究发现, 在上市前一年, VCS 在 DAC 模型和 EM_PROXY 模型中系数显著为正，表明风投机构持股比例越高，风投机构所在上市公司上市前一年进行 应计盈余操控和真实盈余操控的程度越高, 这也反映出在上市前一年风投背景公司存在更高 的应计盈余操控程度和真实盈余操控程度。上市当年, VCS 在 EM_PROXY 模型中系数也同样显 著为正, 进一步验证了本文的研究结论。

以上检验表明，本文的研究结论具有稳健性。

\section{七、结论}

本文以我国证券市场 IPO 公司为研究对象，实证研究风险投资持股公司在上市前后不同 阶段的盈余管理特征，研究发现，出于各种利益动机，在上市前一年，有风险投资持股的公 司相对无风险投资持股的公司存在更高的应计盈余操控程度和真实盈余操控程度，上市当年， 有风险投资持股的公司相对无风险投资持股的公司存在更高的真实盈余操控程度，上市后第 一年，有风险投资持股的公司相对无风险投资持股的公司并不存在更高的应计盈余操控程度 或真实盈余操控程度。另外，国内综合实力更强的会计师事务所在公司上市前一年和上市当 年对公司的盈余管理行为并没有起到更好的监督作用, 而国内综合实力更强的券商在公司上 市前后各阶段对公司的盈余管理行为都没有起到更好的监督作用。

根据本文研究结论，监管当局应进一步完善企业会计准则体系，降低利用应计项目进行 
盈余操控的空间，引导上市公司与拟上市公司切实提高财务信息披露质量。应建立健全风险 投资相关法律法规，加强对风险投资的交易监管和信息披露监管，以更好地规范风险投资行 业的未来发展。另外，监管当局也应完善注册会计师行业的法规制度，加强注册会计师行业 的行政监管，提高审计执业质量；改进保荐人制度，促使保荐代表人承担起严格审核和督导 的责任，切实维护投资者的合法权益。

\section{References}

[1] Barry C B, Muscarella C J, Peavy J W, et al. The role of venture capital in the creation of public companies: Evidence from the going-public process. Journal of Financial economics, 1990, 27(2): 447-471.

[2] Morsfield S G, Tan C E L. Do venture capitalists influence the decision to manage earnings in initial public offerings? The Accounting Review, 2006, 81(5): 1119-1150.

[3] Wongsunwai W. The effect of external monitoring on accrual - based and real earnings management:evidence from venture-backed initial public offerings*. Contemporary Accounting Research, 2013, 30(1): 296-324.

[4] Gompers P A. Grandstanding in the venture capital industry. Journal of Financial economics, 1996, 42(1): 133-156.

[5] Cohen D A, Langberg N S. Venture capital financing and the informativeness of earnings. Asia-Pacific Journal of Accounting \& Economics, 2009, 16(2): 171-189.

[6] Xiangyou Chen. The empirical research on venture capital and earnings management of IPO companies. Research on Financial and Economic Issues, 2010 (1): 64-69.

[7] Ning Jia,Dan Li. The Effect of Venture Capital on the Performance of IPO Companies. Nankai Business Review, 2011 (1): 96-106.

[8] Li Zhang,Yi Tan. The effect of Venture Capital Syndicate on earnings management of IPO firms: based on empirical study of GEM. Journal of Quantitative Economics, 2014, 31(1): 41-47.

[9] Ning Cai. Grandstanding and Earnings Management: Evidence from Venture Capital Industry in China. Accounting Research, 2015, 5: 003. 